Paper No: 19PESGM2082

\title{
GNREL

\section{Data-Enhanced Hierarchical Control to Improve Distribution Voltage with Extremely High PV Penetration}

\author{
Fei Ding, Senior Researcher \\ National Renewable Energy Laboratory \\ Fei.Ding@nrel.gov \\ 2019 IEEE PES General Meeting \\ Atlanta, Georgia \\ August 4-8, 2019
}




\section{Background}

- Develop, validate and deploy Data-Enhanced Hierarchical Control (DEHC) architecture that addresses the challenges associated with high penetration of distributed PV.

- A hierarchical architecture where a fast-acting layer of control interacts with a centralized ADMS system is one of the unique and innovative aspects of this project.

- Offers a unique approach to distribution system operation that has not been developed, tested, and validated before. 


\section{DEHC Architecture}
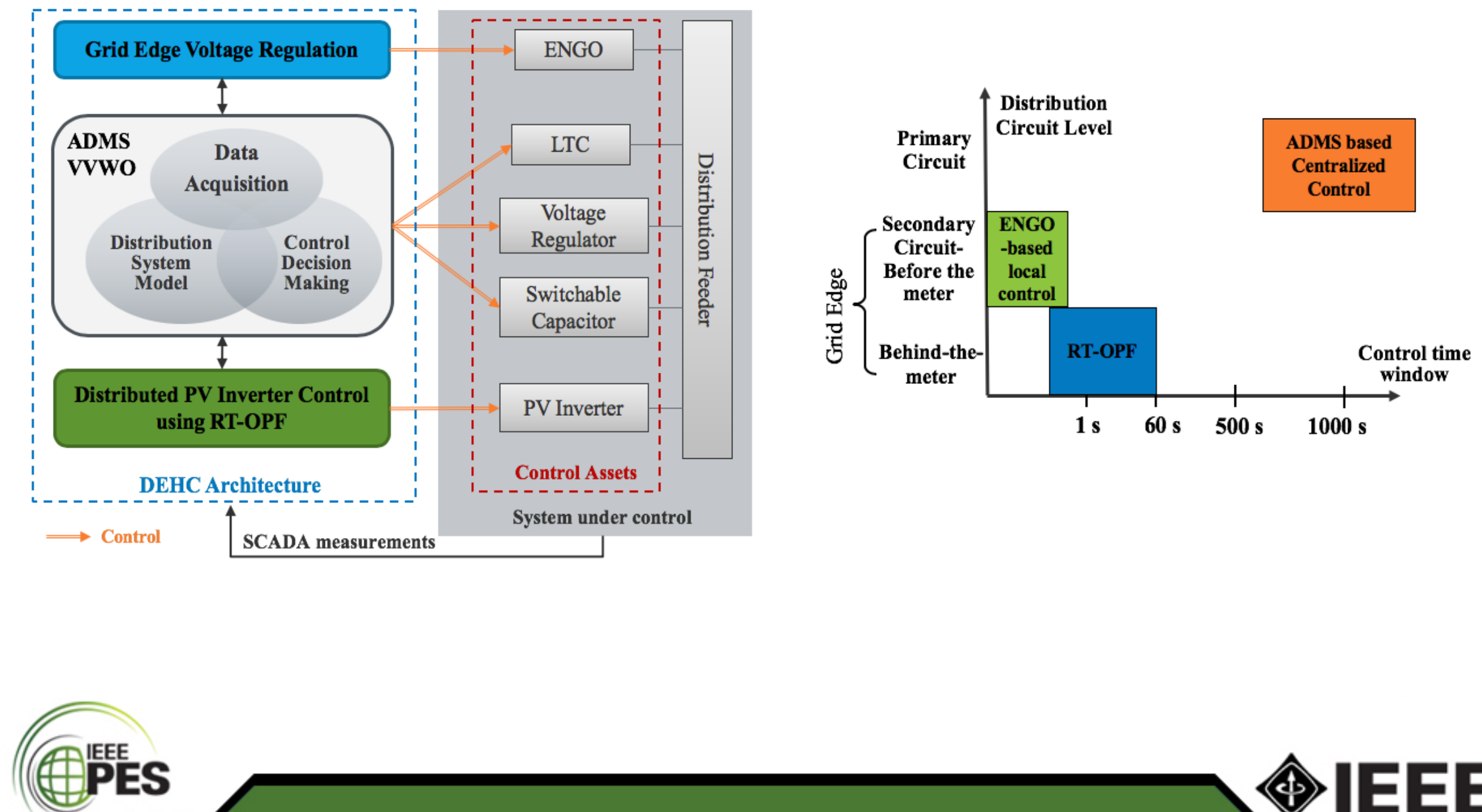

Power \& Energy Society ${ }^{\circ}$ 


\section{Result}
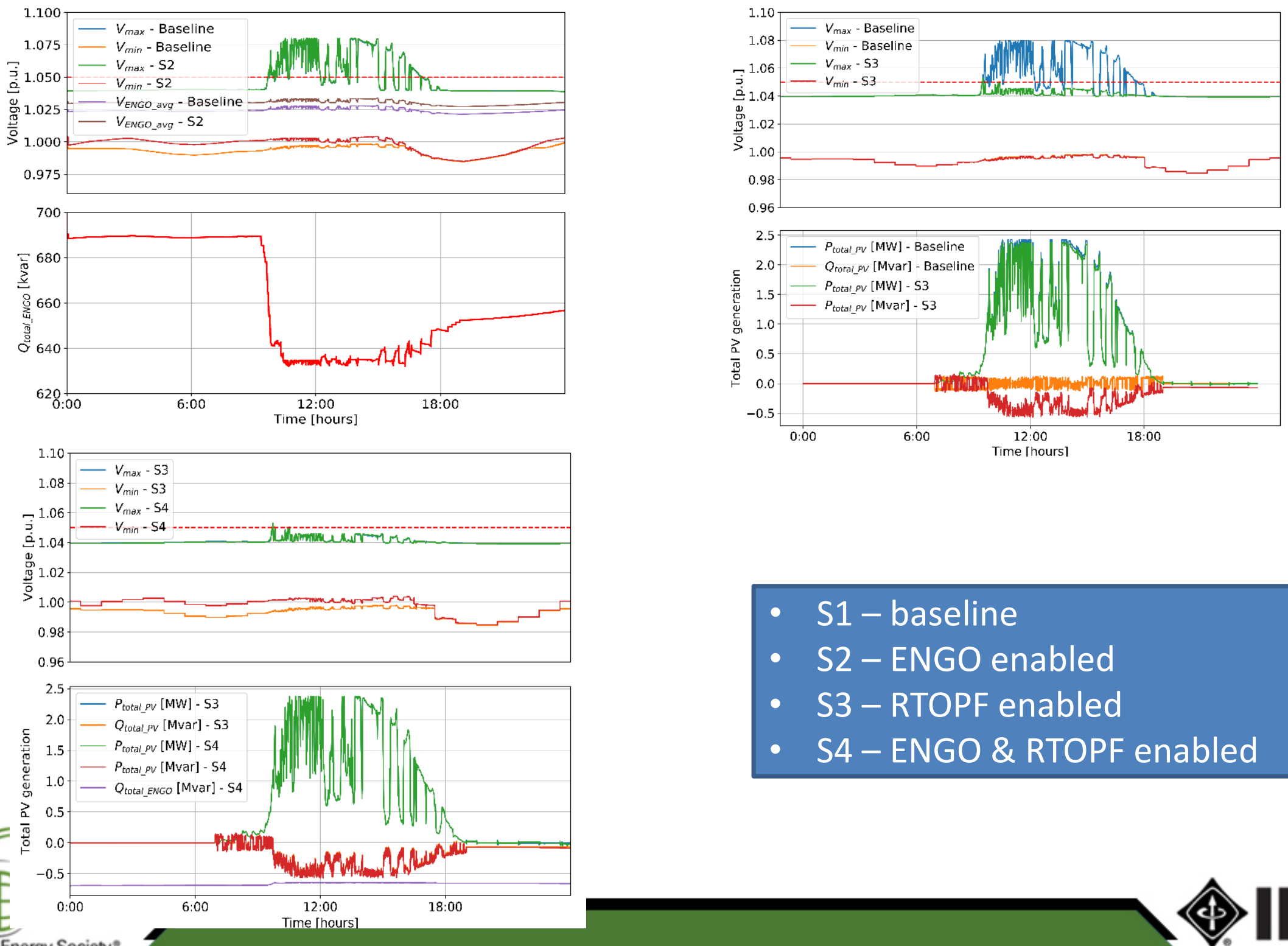

- S1-baseline

- S2 - ENGO enabled

- S3-RTOPF enabled

- S4 - ENGO \& RTOPF enabled 


\section{Thank You!}

This work is funded by the DOE under the Enabling Extreme Real-time Grid Integration of Solar Energy (ENERGISE) program. The authors would like to thank the project partners - EPRI, Schneider Electric, Varentec, and Xcel Energy.

This work was authored in part by the National Renewable Energy Laboratory, operated by Alliance for Sustainable Energy, LLC, for the U.S. Department of Energy (DOE) under Contract No. DE-AC36-08GO28308. Funding provided by U.S. Department of Energy Office of Energy Efficiency and Renewable Energy Solar Energy Technologies Office. The views expressed in the article do not necessarily represent the views of the DOE or the U.S. Government. The U.S. Government retains and the publisher, by accepting the article for publication, acknowledges that the U.S. Government retains a nonexclusive, paid-up, irrevocable, worldwide license to publish or reproduce the published form of this work, or allow others to do so, for U.S. Government purposes. 